International Journal of Bifurcation and Chaos, Vol. 22, No. 7 (2012) 1250176 (12 pages)

(C) World Scientific Publishing Company

DOI: $10.1142 / \mathrm{S} 0218127412501763$

\title{
PINNING IMPULSIVE STABILIZATION OF NONLINEAR DYNAMICAL NETWORKS WITH TIME-VARYING DELAY
}

\author{
JIANQUAN LU \\ Department of Mathematics, Southeast University, \\ Nanjing 210096, P. R. China \\ Potsdam Institute for Climate Impact Research, \\ 14412 Potsdam, Germany \\ jqluma@seu.edu.cn \\ ZIDONG WANG \\ Department of Information Systems and Computing, \\ Brunel University, Uxbridge, Middlesex, UB8 3PH, UK \\ Zidong.Wang@brunel.ac.uk \\ JINDE CAO \\ Department of Mathematics, Southeast University, \\ Nanjing 210096, P. R. China \\ jdcao@seu.edu.cn \\ DANIEL W.C. HO \\ Department of Mathematics, \\ City University of Hong Kong, Hong Kong \\ madaniel@cityu.edu.hk \\ JÜRGEN KURTHS \\ Potsdam Institute for Climate Impact Research, \\ 14412 Potsdam, Germany \\ Department of Physics, Humboldt University Berlin, \\ 12489 Berlin, Germany \\ Juergen.Kurths@pik-potsdam.de
}

Received October 31, 2011; Revised March 8, 2012

\begin{abstract}
In this paper, a new impulsive control strategy, namely pinning impulsive control, is proposed for the stabilization problem of nonlinear dynamical networks with time-varying delay. In this strategy, only a small fraction of nodes is impulsively controlled to globally exponentially stabilize the whole dynamical network. By employing the Lyapunov method combined with the mathematical analysis approach as well as the comparison principle for impulsive systems, some criteria are obtained to guarantee the success of the global exponential stabilization process. The obtained criteria are closely related to the proportion of the controlled nodes, the impulsive strength, the impulsive interval and the time-delay. Numerical examples are given to demonstrate the effectiveness of the designed pinning impulsive controllers.
\end{abstract}

Keywords: Pinning impulsive control; global exponential synchronization; complex networks; time-varying delay. 


\section{Introduction}

Recently, increasing interest has been devoted to the study of collective behaviors in complex dynamical networks due to its frequently occurrence in natural systems and widely potential applications [Strogatz, 2001; Zhou et al., 2006; Gao et al., 2006; Wang et al., 2010; Motter et al., 2005; Wang \& Chen, 2002]. Some recent advances on network synchronization have been reported in [Arenas et al., 2008].

A complex dynamical network is a large set of interconnected nodes, and each node can be a nonlinear dynamical system with chaotic, periodic or stable behavior. Synchronization, which means that the state variables of the individual nodes converge towards each other, can be realized by node information being exchanged via inter-connections [Wu, 2007; Chen et al., 2004]. However, the final synchronous state of the network, which is achieved by the interconnecting coupling of the nodes, is very difficult to estimate and predict due to the individuals' active dynamics. There is a common need to regulate the behavior of large ensembles of interacting individuals by external forces for many biological, physical and social dynamical networks [Mazenc et al., 2008; Lu et al., 2008]. For example, many regulatory mechanisms have been uncovered in the context of physiological, biological and cellular processes to control the dynamical processes so as to evolve towards a desired structure, which are fundamental to guarantee the correct functioning of the whole network [Mazenc et al., 2008]. Control methods have also been regarded as one of the four key features to deeply understand biological systems at a system level [Kitano, 2002]. The issue of consensus of multiagent systems [Olfati-Saber \& Murray, 2004; Lu et al., 2009a] is closely related to the synchronization problem on dynamical networks. This is also another very interesting collective behavior that is of great interest to researchers in computer science, biological systems, ecosystems and several other areas [Solé \& Bascompte, 2006].

Various control strategies including adaptive control [Lu et al., 2008; Zhang et al., 2008; Wang et al., 2008; Gleiser \& Zanette, 2006], impulsive control [Liu et al., 2005; Guan et al., 2010; Zhang et al., 2010; Tang et al., 2010] and pinning statefeedback control [Li et al., 2004; Chen et al., 2007; Lu et al., 2009b; Yu et al., 2009; Sorrentino et al., 2007; Lu \& Ho, 2011] have been proposed to regulate different kinds of complex dynamical networks with time-delay or noise. Pinning state-feedback control here means that only a small fraction of nodes is selected to be controlled to stabilize the whole dynamical network. A great number of results have been published in the recent literature on the general topic of pinning state-feedback control for network synchronization, most of which are essentially obtained based on the fact that the largest eigenvalue of the coupling matrix can be changed from zero to be negative via state-feedback pinning [Chen et al., 2007; Lu et al., 2009c]. Adaptive controllers and impulsive controllers are simultaneously used to synchronize a stochastic coupled network in [Tang et al., 2011]. Pinning control makes the network control more convenient to implement since only a small fraction of nodes is directly controlled. On the other hand, it is well-known that the impulsive control allows the stabilization of dynamical networks and chaotic systems using only small control impulses, it has been widely used to stabilize dynamical networks and chaotic systems [Yang, 2001; Liu \& Zhao, 2011]. However, impulsive control is not easy to implement in a complex network environment if all nodes have to be controlled as in [Liu et al., 2005; Guan et al., 2010; Zhang et al., 2010; Tang et al., 2010]. Unfortunately, to the best of our knowledge, pinning impulsive control for network stabilization, though vitally important for understanding network stabilization processes and impulsive feature, has not yet been studied primarily due to the mathematical difficulties.

It is noted that time delays are often encountered in real world [Cao et al., 2008; Ponce et al., 2009], and the delays are usually time-varying in electronic implementation of analog networks due to the finite switching speed of amplifiers. In many cases, only partial information about the timevarying delay is known such as the upper bound, and the time-varying delay can be even nonsmooth. Design flaws and incorrect analytical conclusions can be obtained if time-varying delays are not considered or not well described in system modeling. Therefore, it is of great importance to consider the effects of time-varying delay for the pinning impulsive stabilization of dynamical networks.

Motivated by the above discussions, we aim to study the pinning stabilization problem of nonlinear complex dynamical networks with time-varying delay. Instead of state-feedback controllers, impulsive controllers will be used to pin a small fraction of nodes for successful control, and the states of 
the whole dynamical network can be globally exponentially forced to the objective state. The nodes to be impulsively controlled should be different at distinct control instants. Some easily-verified criteria will be presented by employing some analytical methods, Lyapunov method and comparison principle for impulsive systems. The relationship among the proportion of the controlled nodes, an upper bound of time-varying delay, impulsive strengths, and impulsive interval of the dynamical networks is well explained in the obtained criteria. Simulation examples are given to demonstrate the effectiveness of the proposed pinning impulsive method.

The rest of this paper is organized as follows. In Sec. 2, some preliminaries are presented. In Sec. 3, pinning impulsive control strategy is given and some stabilization criteria are derived. In Sec. 4, numerical examples are given to demonstrate the effectiveness of the results obtained. Concluding remarks are drawn in Sec. 5.

Notations. The standard notations will be used in this paper. $I_{n}$ is the identity matrix of order $n$. We use $\lambda_{\max }(\cdot)$ to denote the maximum eigenvalue of a real symmetric matrix. $\mathbb{R}^{n}$ denotes the $n$-dimensional Euclidean space. $\mathbf{0}$ denotes $n$-dimensional zero vector. $\mathbb{R}^{n \times n}$ are $n \times n$ real matrices. The superscript " $T$ " represents the transpose. $\operatorname{diag}\{\cdots\}$ stands for a block-diagonal matrix. Matrices, if not explicitly stated, are assumed to have compatible dimensions. Let $\|x\|$ be the Euclid vector norm of $x \in \mathbb{R}^{n}$, and $\mathbb{P} \mathbb{C}(m)$ denote the class of piecewise right continuous function $\varphi:\left[t_{0}-\tau,+\infty\right) \rightarrow \mathbb{R}^{m}(m \in \mathbb{N})$ with the norm defined by $\|\varphi(t)\|_{\tau}=\sup _{-\tau<s \leq 0}\|\varphi(t+s)\|$. For $\varphi: \mathbb{R} \rightarrow \mathbb{R}$, denote $\varphi\left(t^{+}\right)=\lim _{s \rightarrow 0^{+}} \varphi(t+s)$ and $\varphi\left(t^{-}\right)=\lim _{s \rightarrow 0^{-}} \varphi(t+s)$. \#G denotes the number of elements of a finite set $G$. The Dini derivative of $\varphi(t)$ is defined as $D^{+} \varphi(t)=\lim \sup _{s \rightarrow 0^{+}} \frac{\varphi(t+s)-\varphi(t)}{s}$.

\section{Some Preliminaries}

In this section, some preliminaries including model formulation and some lemmas are presented. The basic nonlinear dynamical network model will be introduced in various stages starting with a trivial network of decoupled nodes governed by delayed differential equations.

A neural network is a mathematical model consisting of an interconnected group of artificial neurons, which has potential applications in secure communication and pattern recognition [Fischer et al., 2000]. In this paper, the following neural network with time-varying delay [Cao \& Ho, 2005] is taken as a single node of the nonlinear dynamical network:

$$
\begin{aligned}
\dot{y}(t)= & -C y(t)+B \tilde{f}_{1}(y(t)) \\
& +D \tilde{f}_{2}(y(t-\tau(t)))
\end{aligned}
$$

where $y(t)=\left(y_{1}(t), y_{2}(t), \ldots, y_{n}(t)\right)^{T} \in \mathbb{R}^{n}$ is the state vector associated with the neurons; $C=$ $\operatorname{diag}\left(c_{1}, c_{2}, \ldots, c_{n}\right)>0$ (a positive-definite diagonal matrix), $B=\left(b_{i j}\right)_{n \times n}$ and $D=\left(d_{i j}\right)_{n \times n}$ are the connection weight matrix and the delayed connection matrix, respectively; $\tau(t)$ is the transmission time-varying delay satisfying $0<\tau(t) \leq \tau^{*}$; $\tilde{f}_{1}(y(t))=\left(\tilde{f}_{11}\left(y_{1}(t)\right), \ldots, \tilde{f}_{1 n}\left(y_{n}(t)\right)\right)^{T} \in \mathbb{R}^{n}$ and $\tilde{f}_{2}(y(t-\tau(t)))=\left(\tilde{f}_{21}\left(y_{1}(t-\tau(t)), \ldots, \tilde{f}_{2 n}\left(y_{n}(t-\right.\right.\right.$ $\tau(t))))^{T} \in \mathbb{R}^{n}$ denote the activation functions of the neurons satisfying $\tilde{f}_{1}(\mathbf{0})=\mathbf{0}$ and $\tilde{f}_{2}(\mathbf{0})=\mathbf{0}$.

By nonlinear coupling of $N$ nodes in a complex network, we can obtain the following nonlinear dynamical network model:

$$
\begin{aligned}
\dot{x}_{i}(t)= & -C x_{i}(t)+B \tilde{f}_{1}\left(x_{i}(t)\right)+D \tilde{f}_{2}\left(x_{i}(t-\tau(t))\right) \\
& +c \sum_{j=1}^{N} a_{i j} \Gamma \tilde{H}\left(x_{j}(t)\right), \quad i=1,2, \ldots, N,
\end{aligned}
$$

where $x_{i}(t) \in \mathbb{R}^{n}$ is the state vector of the $i$ th node; $\Gamma=\operatorname{diag}\left\{\gamma_{1}, \gamma_{2}, \ldots, \gamma_{n}\right\}>0$ is the inner coupling matrix between two connected nodes; $c>0$ is the coupling strength of the dynamical network; the nonlinear function is defined as $\tilde{H}\left(x_{j}(t)\right)=\left(\tilde{h}\left(x_{j 1}(t)\right), \tilde{h}\left(x_{j 2}(t)\right), \ldots, \tilde{h}\left(x_{j n}(t)\right)\right)^{T}$ and $\tilde{h}(\cdot)$ is a monotonically increasing function satisfying $[(\tilde{h}(u)-\tilde{h}(v)) /(u-v)] \geq \vartheta>0$ for any $u, v \in$ $\mathbb{R} ; A=\left(a_{i j}\right)_{N \times N}$ is the Laplacian coupling matrix representing the coupling topology of the dynamical network [Chung, 1997]. The elements $a_{i j}$ of the matrix $A$ are defined as follows: if there is a connection between nodes $i$ and $j(i \neq j), a_{i j}=a_{j i}>0$; otherwise $a_{i j}=a_{j i}=0(i \neq j)$, and the diffusivity coupling condition $a_{i i}=-\sum_{j=1, j \neq i}^{N} a_{i j}$ is satisfied for the diagonal elements $a_{i i}(i=1,2, \ldots, N)$. The Laplacian matrix $A$ is assumed to be irreducible throughout this paper. This diffusivity condition implies that the nonlinearly coupled dynamical network would be decoupled when the array of nodes is synchronized.

In this paper, the objective trajectory that the nonlinear dynamical network (2) will be forced to, 
is selected as any solution $s(t)$ of the system (1) satisfying $\dot{s}(t)=-C s(t)+B \tilde{f}_{1}(s(t))+D \tilde{f}_{2}(s(t-\tau(t)))$. Let $e_{i}(t)=x_{i}(t)-s(t)$ be the error state of node $i$ between the current state $x_{i}(t)$ and the objective state $s(t)$. Then, the following error dynamical network can be obtained by considering the fact that $c \sum_{j=1}^{N} a_{i j} \Gamma \tilde{H}(s(t))=0$ :

$$
\begin{aligned}
\dot{e}_{i}(t)= & -C e_{i}(t)+B f_{1}\left(e_{i}(t)\right)+D f_{2}\left(e_{i}(t-\tau(t))\right) \\
& +c \sum_{j=1}^{N} a_{i j} \Gamma H\left(e_{j}(t)\right), \quad i=1,2, \ldots, N
\end{aligned}
$$

where $f_{1}\left(e_{i}(t)\right)=\tilde{f}_{1}\left(x_{i}(t)\right)-\tilde{f}_{1}(s(t)), f_{2}\left(e_{i}(t)\right)=$ $\tilde{f}_{2}\left(x_{i}(t)\right)-\tilde{f}_{2}(s(t))$, and $H\left(e_{j}(t)\right)=\tilde{H}\left(x_{i}(t)\right)-$ $\left.\tilde{H}(s(t)):=\left(h\left(e_{j 1}(t)\right), h\left(e_{j 2}(t)\right), \ldots, h\left(e_{j n}(t)\right)\right)^{T}\right)$. By referring to the property of the function $\tilde{H}(\cdot)$, we have $[(h(u)-h(v)) /(u-v)] \geq \vartheta>0$.

The following assumption is needed for the proof of the main results in the next section.

Assumption 1. The neuron activation functions $\tilde{f}_{1}(\cdot)$ and $\tilde{f}_{2}(\cdot)$ of the neural network satisfy the following Lipschitz condition:

$$
\begin{aligned}
& \left\|\tilde{f}_{1}(x)-\tilde{f}_{1}(y)\right\| \leq l_{1}\|x-y\|, \quad \forall x, y \in \mathbb{R}^{n}, \\
& \left\|\tilde{f}_{2}(x)-\tilde{f}_{2}(y)\right\| \leq l_{2}\|x-y\|,
\end{aligned}
$$

where $l_{1}$ and $l_{2}$ are positive constants.

\section{Main Results}

In this section, pinning impulsive controllers will be designed to globally and exponentially stabilize the dynamical network with nonlinear coupling. In order to drive the nonlinear dynamical network into the objective state $s(t)$, the following impulsive controllers are constructed for $l$ nodes $(l \ll N)$ :

$$
u_{i}(t)=\sum_{k=1}^{+\infty} \mu e_{i}(t) \delta\left(t-t_{k}\right), \quad i \in \mathfrak{D}(t),
$$

where $\mu \in(-2,-1) \cup(-1,0)$ is a constant, which means that the impulsive effects can be used to stabilize the nonlinear dynamical network; the time series $\left\{t_{1}, t_{2}, t_{3}, \ldots\right\}$ is a sequence of strictly increasing impulsive instants satisfying $\lim _{k \rightarrow \infty} t_{k}=\infty$, and the index set of $l$ nodes $\mathfrak{D}(t)$ which should be impulsively controlled is defined as follows: At time instant $t$, for the error states $e_{1}(t), e_{2}(t), \ldots, e_{N}(t)$, one can reorder the vectors such that $\left\|e_{p 1}(t)\right\| \geq$ $\left\|e_{p 2}(t)\right\| \geq \cdots \geq\left\|e_{p l}(t)\right\| \geq \cdots \geq\left\|e_{p N}(t)\right\|$. Then, the index set of $l$ controlled nodes $\mathfrak{D}(t)$ is defined as $\mathfrak{D}(t)=\left\{p_{1}, p_{2}, \ldots, p_{l}\right\}$.

Remark 3.1. If there are more than $l$ states with the same norm (i.e. $\left\|e_{p l}(t)\right\|=\left\|e_{p, l+1}(t)\right\|=\cdots=$ $\left.\left\|e_{p, l+\varpi}(t)\right\|\right)$, any node with the same norm can be selected into the set $\mathfrak{D}(t)$ for satisfying $\# \mathfrak{D}(t)=l$.

After adding the pinning impulsive controllers (5) to the nodes $\mathfrak{D}(t)$, the controlled dynamical network can be rewritten as follows:

$$
\left\{\begin{aligned}
& \dot{e}_{i}(t)=-C e_{i}(t)+B f_{1}\left(e_{i}(t)\right) \\
& \\
&+D f_{2}\left(e_{i}(t-\tau(t))\right)+c \sum_{j=1}^{N} a_{i j} \Gamma H\left(e_{j}(t)\right), \\
& t \geq 0, \quad t \neq t_{k}, \quad k \in \mathbb{N}, \\
& e_{i}\left(t_{k}^{+}\right)=e_{i}\left(t_{k}^{-}\right)+\mu e_{i}\left(t_{k}^{-}\right), \quad i \in \mathfrak{D}\left(t_{k}\right) .
\end{aligned}\right.
$$

Remark 3.2. From the second equation of (6), we can see that only $l(l \ll N)$ nodes are impulsively controlled. This means that we do not need to add controllers to each node, and hence the control cost can be substantially lower and our strategy can be easier to implement. It is noted that the controlled nodes can be distinct at different instants.

The initial conditions of dynamical network (6) are given by

$$
e_{i}(t)=\phi_{i}(t), \quad-\tau^{*} \leq t \leq 0, \quad i=1,2, \ldots, N,
$$

where $\phi_{i}(t) \in \mathbb{C}\left(\left[-\tau^{*}, 0\right], \mathbb{R}^{n}\right)$ is the set of continuous functions from $\left[-\tau^{*}, 0\right]$ to $\mathbb{R}^{n}$.

Throughout this paper, we always assume that $e_{i}(t)$ is right continuous at $t=t_{k}$, i.e. $e\left(t_{k}\right)=e\left(t_{k}^{+}\right)$. Therefore, the solutions of (6) are piecewise righthand continuous functions with discontinuities at $t=t_{k}$ for $k \in \mathbb{N}$.

Definition 3.1. The nonlinear dynamical network (2) is said to be globally exponentially stabilized to the objective state $s(t)$ if there exist $\lambda>0$, $T_{0}>0$ and $\theta>0$ such that for any initial values $\phi_{i}(\cdot)(i=1,2, \ldots, N)$,

$$
\left\|e_{i}(t)\right\|=\left\|x_{i}(t)-s(t)\right\| \leq \theta e^{-\lambda t}
$$

hold for all $t>T_{0}$, and for any $i=1,2, \ldots, N$. 
Lemma 1 [Yang \& Xu, 2007]. Let $0 \leq \tau_{i}(t) \leq \tau$, $F\left(t, u, \bar{u}_{1}, \ldots, \bar{u}_{m}\right) \quad: \quad \mathbb{R}^{+} \times \overbrace{\mathbb{R} \times \cdots \times \mathbb{R}}^{m+1} \rightarrow \mathbb{R}$ be nondecreasing in $\bar{u}_{i}$ for each fixed $\left(t, u, \bar{u}_{1}, \ldots, \bar{u}_{i-1}, \bar{u}_{i+1}, \ldots, \bar{u}_{m}\right), \quad i=1,2, \ldots, m$, and $I_{k}(u): \mathbb{R} \rightarrow \mathbb{R}$ be nondecreasing in u. Suppose that $u(t), v(t) \in \mathbb{P} \mathbb{C}(1)$ satisfy

$$
\left\{\begin{array}{c}
\begin{array}{rl}
D^{+} u(t) \leq F\left(t, u(t), u\left(t-\tau_{1}(t)\right), \ldots,\right. \\
& \left.u\left(t-\tau_{m}(t)\right)\right), \quad t \neq t_{k}, \quad t \geq 0 \\
u\left(t_{k}\right) \leq I_{k}\left(u\left(t_{k}^{-}\right)\right), \quad k \in \mathbb{N}
\end{array}
\end{array}\right.
$$

and

$$
\left\{\begin{aligned}
& D^{+} v(t)> F\left(t, v(t), v\left(t-\tau_{1}(t)\right), \ldots\right. \\
&\left.v\left(t-\tau_{m}(t)\right)\right), \quad t \neq t_{k}, \quad t \geq 0 \\
& v\left(t_{k}\right) \geq I_{k}\left(v\left(t_{k}^{-}\right)\right), \quad k \in \mathbb{N} .
\end{aligned}\right.
$$

Then $u(t) \leq v(t)$, for $-\tau \leq t \leq 0$ implies $u(t) \leq$ $v(t)$, for $t \geq 0$.

Remark 3.3. Lemma 1 is an extended comparison theorem for the impulsive system with timedelay. By using the comparison principle and the Lyapunov stability method [Lu et al., 2011; Lu et al., 2010; Yang \& Xu, 2007], the main result on pinning impulsive stabilization of nonlinear dynamical networks will be derived.

Theorem 1. Let $\rho=\frac{N+l \mu(\mu+2)}{N} \in(0,1)$ and $T=$ $\max _{k \in \mathbb{N}}\left\{t_{k+1}-t_{k}\right\}<\infty$. Suppose that

$$
\begin{array}{r}
\lambda_{\max }\left(-C-C^{T}\right)+2 l_{1} \sqrt{\lambda_{\max }\left(B^{T} B\right)} \\
+2 l_{2} \sqrt{\frac{\lambda_{\max }\left(D^{T} D\right)}{\rho}}+\frac{\ln \rho}{T}<0,
\end{array}
$$

then the complex dynamical network (6) with pinning impulsive controllers is globally exponentially stable in the following sense:

$$
\left\|e_{i}(t)\right\| \leq \rho^{-1} \sup _{-\tau^{*} \leq s \leq 0}\left\{\sum_{i=1}^{N}\left\|\phi_{i}(s)\right\|^{2}\right\} e^{-\lambda t}
$$

where $\lambda>0$ is a unique solution of

$$
\lambda-\beta+\rho^{-\frac{1}{2}} d \cdot e^{\lambda \tau^{*}}=0, \quad t>0
$$

in which

$$
\begin{aligned}
\beta= & -\left(\lambda_{\max }\left(-C-C^{T}\right)\right. \\
& \left.+2 l_{1} \sqrt{\lambda_{\max }\left(B^{T} B\right)}+\frac{d}{\sqrt{\rho}}+\frac{\ln \rho}{T}\right)
\end{aligned}
$$

and $d=l_{2} \sqrt{\lambda_{\max }\left(D^{T} D\right)}$. This means that the nonlinear dynamical network (2) can be exponentially stabilized to the objective state $s(t)$ by the pinning impulsive controllers (5) if the inequality (8) is satisfied.

Remark 3.4. In Theorem $1, T=\max _{k \in \mathbb{N}}\left\{t_{k+1}-\right.$ $\left.t_{k}\right\}$ is the maximum impulsive interval which means the maximum time difference between two impulses. Normally, this concept is used, when the impulses are synchronizing (or stabilizing), in order to characterize the frequency of impulses and to guarantee that the frequency of impulses should not be too low. By revising the proof of the main results, a newly proposed concept of "average impulsive interval" [Lu et al., 2010] can be used to make the obtained result less conservative.

Remark 3.5. Although the coupling matrix $A$ in this paper is assumed to be irreducible, the results in Theorem 1 can be easily extended to the case of a reducible coupling matrix [Lu et al., 2009b] by utilizing the normalized left eigenvector of the coupling matrix with respect to the eigenvalue zero and using the fact that the maximum value of the real part of the eigenvalue is zero. For directed networks with a rooted spanning tree, it would be much easier to control the whole network by impulsively controlling a small fraction of nodes in the group of rooted nodes and the nonrooted nodes can be virtually controlled.

For linearly coupled dynamical networks without delay term (i.e. $\tilde{h}\left(x_{j}(t)\right)=x_{j}(t)$ and $D=\mathbf{0}$ ), one can easily derive the following corollary. Consider the following linearly coupled dynamical networks:

$$
\begin{aligned}
\dot{x}_{i}(t)= & -C x_{i}(t)+B \tilde{f}_{1}\left(x_{i}(t)\right) \\
& +c \sum_{j=1}^{N} a_{i j} \Gamma x_{j}(t), \quad i=1,2, \ldots, N .
\end{aligned}
$$


Corollary 3.1. Let $\rho=\frac{N+l \mu(\mu+2)}{N} \in(0,1)$ and $T=\max _{k \in \mathbb{N}}\left\{t_{k}-t_{k-1}\right\}<\infty$. Suppose that

$$
\lambda_{\max }\left(-C-C^{T}\right)+2 l_{1} \sqrt{\lambda_{\max }\left(B^{T} B\right)}+\frac{\ln \rho}{T}<0,
$$

then the linearly coupled dynamical network (11) can be exponentially forced onto the objective trajectory $s(t)$ by pinning impulsive controllers (5) with the convergence rate $\lambda=-\left(\lambda_{\max }\left(-C-C^{T}\right)+\right.$ $\left.2 l_{1} \sqrt{\lambda_{\max }\left(B^{T} B\right)}+\frac{\ln \rho}{T}\right)$.

Remark 3.6. It can be observed from Theorem 1 and Corollary 3.1 that whether the pinning impulsive control and the stabilizing convergent rate can be successful depends on the proportion of the controlled nodes, the upper bound of the time-varying delay, the impulsive strengths and the impulsive interval of the dynamical networks. The higher the proportion of the controlled nodes is, the easier the dynamical network can be efficiently controlled.

\section{Numerical Examples}

In this section, two examples are shown to illustrate the results obtained in the previous section. Consider the following system as the individual node in the dynamical network:

$$
\begin{aligned}
\dot{y}(t)= & -C y(t)+B \tilde{f}_{1}(y(t)) \\
& +D \tilde{f}_{2}(y(t-\tau(t))),
\end{aligned}
$$

with $C=I_{2}, B=\left(\begin{array}{rc}2.0 & -0.11 \\ -5.0 & 3.2\end{array}\right), D=\left(\begin{array}{ll}-1.6 & -0.1 \\ -0.18 & -2.4\end{array}\right)$, and $\tau(t)=\frac{e^{t}}{1+e^{t}} \leq 1$, where $y(t)=\left(y_{1}(t), y_{2}(t)\right)^{T}$ is the state vector of the single neural network, and $\tilde{f}_{1}(y(t))=\tilde{f}_{2}(y(t))=\left(\tanh \left(y_{1}\right), \tanh \left(y_{2}\right)\right)^{T}$. Then, one can easily obtain $l_{1}=l_{2}=1$ for satisfying Assumption 1. The single individual (13) has a chaotic attractor [Lu, 2002] (as shown in Fig. 1) for the initial values $y_{1}(\varrho)=0.2, y_{2}(\varrho)=0.5$, $\forall \varrho \in[-1,0]$.

As examples, two practical network models including BA (Barabási-Albert) scale-free network [Barabási \& Albert, 1999] and NW (NewmanWatts) small-world network [Newman \& Watts, 1999] are considered. A 100 nodes' scale-free network is generated by taking $m=m_{0}=5$. A 100 nodes' small-world network is generated by taking initial neighboring nodes $k=4$ and the edge adding probability $p=0.1$. The weight of the coupling matrix $A$ is defined as follows: if there is a connection between nodes $i$ and $j$, then $a_{i j}=a_{j i}=1$, otherwise $a_{i j}=a_{j i}=0$.

The parameters corresponding to the network coupling are taken as follows: the nonlinear coupling function $\tilde{h}$ is taken as $\tilde{h}(z)=z+\tanh (z)$ with $\vartheta=1$, coupling strength $c=2$ and the inner coupling matrix $\Gamma=I_{2}$.

In both examples, according to the algorithm described before Remark $3.1, l=30$ nodes are selected to be pinning controlled at each time instant, and the objective trajectory $s(t)$ is taken as $s(t)=0$. Take $\mu=-0.9$ and $t_{k}-t_{k-1}=0.02$,

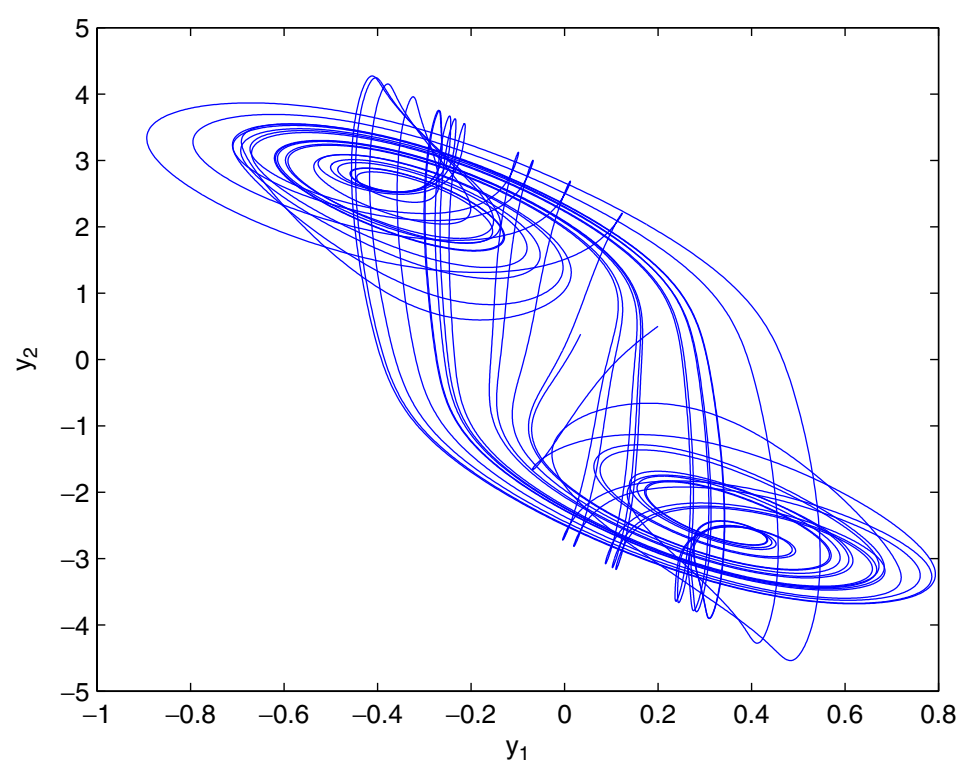

Fig. 1. Chaotic attractor of the single individual (13) in the dynamical network. 


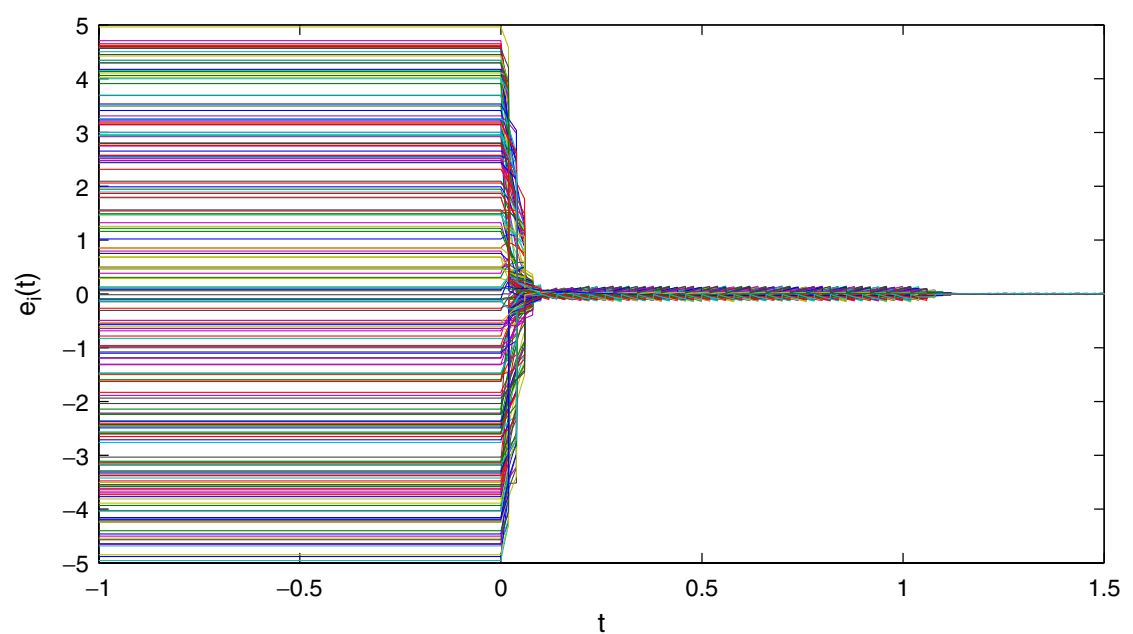

Fig. 2. The states of 100 nodes' scale-free network are successfully forced to the objective state $(s(t)=0)$ by impulsively controlling 30 nodes.

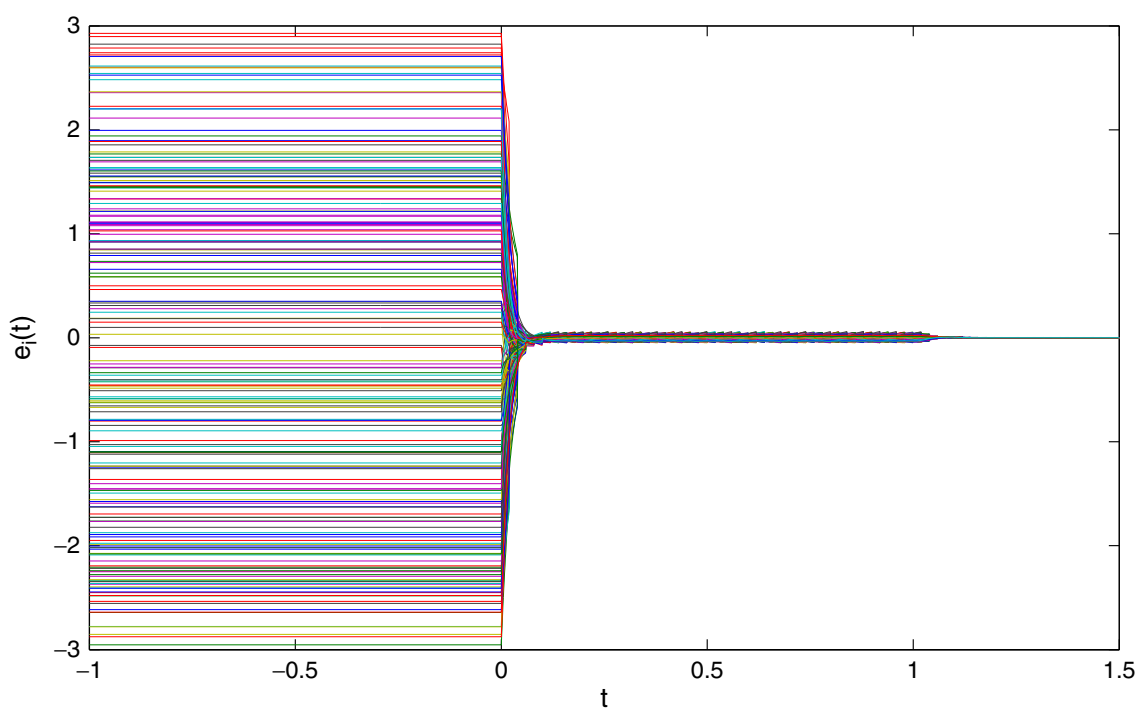

Fig. 3. The 100 nodes' states in small-world network are forced to the objective original state by impulsively controlling 30 nodes.

one gets that $\lambda_{\max }\left(-C-C^{T}\right)+2 l_{1} \sqrt{\lambda_{\max }\left(B^{T} B\right)}+$ $2 l_{2} \sqrt{\frac{\lambda_{\max }\left(D^{T} D\right)}{\rho}}+\frac{\ln \rho}{T}=-1.4508<0$. According to Theorem 1, we can conclude that both networks can be successfully stabilized to the objective trajectory $s(t)$. Figures 2 and 3 are plotted to present the successful stabilization process, respectively, for scale-free and small-world networks. In the simulations, all initial conditions of the nodes are randomly selected from $[-5,5]$.

\section{Conclusion}

This paper has been devoted to studying pinning impulsive stabilization of complex dynamical networks with nonlinear coupling and time-varying delay. Different from existing results about impulsive control, only a small fraction of nodes has been selected to be impulsively controlled to stabilize the whole state-coupled dynamical networks. By using an impulsive delay differential inequality and an efficient algorithm, a small fraction of nodes has been carefully chosen to be controlled by designed impulsive controllers. The derived stabilization criterion and the convergence rate are closely related with the proportion of the controlled nodes, the time-delay, impulsive strengths, and impulsive interval of the dynamical networks. There is no more constraint on the time-varying delay 
except boundedness. Two simulation examples have been presented to illustrate the usefulness and effectiveness of the main results obtained.

\section{Acknowledgments}

The work of J. Q. Lu was supported by the National Natural Science Foundation of China (NSFC) under Grant 61175119, the Natural Science Foundation of Jiangsu Province of China under Grant BK2010408, Huo Ying-Dong Foundation (Grant No. 132037), and the Alexander von Humboldt Foundation of Germany. The work of J. D. Cao was supported by the National Natural Science Foundation of China under Grant No. 11072059, and the Specialized Research Fund for the Doctoral Program of Higher Education under Grant No. 20110092110017. This work of D. W. C. Ho was supported by a grant from CityU (7002561) and a GRF grant by HKSAR (9041432). The work of J. Kurths was supported by SUMO (EU), GSDP (EU) and IRTG 1740 (DFG).

\section{References}

Arenas, A., Díaz-Guilera, A., Kurths, J., Moreno, Y. \& Zhou, C. [2008] "Synchronization in complex networks," Phys. Rep. 469, 93-153.

Barabási, A. \& Albert, R. [1999] "Emergence of scaling in random networks," Science 286, 509-512.

Cao, J. \& Ho, D. [2005] "A general framework for global asymptotic stability analysis of delayed neural networks based on LMI approach," Chaos Solit. Fract. 24, 1317-1329.

Cao, J., Chen, G. \& Li, P. [2008] "Global synchronization in an array of delayed neural networks with hybrid coupling," IEEE Trans. Syst. Man Cybern. Part B 38, 488-498.

Chen, G., Zhou, J. \& Liu, Z. [2004] "Global synchronization of coupled delayed neural networks and applications to chaotic CNN models," Int. J. Bifurcation and Chaos 14, 2229-2240.

Chen, T., Liu, X. \& Lu, W. [2007] "Pinning complex networks by a single controller," IEEE Trans. Circuits Syst.-I 54, 1317-1326.

Chung, F. [1997] Spectral Graph Theory (American Mathematical Society).

Fischer, I., Liu, Y. \& Davis, P. [2000] "Synchronization of chaotic semiconductor laser dynamics on subnanosecond time scales and its potential for chaos communication," Phys. Rev. A 62, 011801.

Gao, H., Lam, J. \& Chen, G. [2006] "New criteria for synchronization stability of general complex dynamical networks with coupling delays," Phys. Lett. A 360, 263-273.
Gleiser, P. \& Zanette, D. [2006] "Synchronization and structure in an adaptive oscillator network," The Europ. Phys. J. B 53, 233-238.

Guan, Z., Liu, Z., Feng, G. \& Wang, Y. [2010] "Synchronization of complex dynamical networks with time-varying delays via impulsive distributed control," IEEE Trans. Circuits Syst.-I 57, 2182-2195.

Kitano, H. [2002] "Systems biology: A brief overview," Science 295, 1662-1664.

Lakshmikantham, V., Bainov, D. \& Simeonov, P. [1989] Theory of Impulsive Differential Equations (World Scientific, Singapore).

Li, X., Wang, X. \& Chen, G. [2004] "Pinning a complex dynamical network to its equilibrium," IEEE Trans. Circuits Syst.-I 51, 2074-2087.

Liu, B., Liu, X., Chen, G. \& Wang, H. [2005] "Robust impulsive synchronization of uncertain dynamical networks," IEEE Trans. Circuits Syst.-I 52, 1431-1441.

Liu, Y. \& Zhao, S. [2011] "Controllability for a class of linear time-varying impulsive systems with time delay in control input," IEEE Trans. Autom. Contr. 99, 395-399.

Lu, H. [2002] "Chaotic attractors in delayed neural networks," Phys. Lett. A 298, 109-116.

Lu, J., Ho, D. \& Cao, J. [2008] "Synchronization in an array of nonlinearly coupled chaotic neural networks with delay coupling," Int. J. Bifurcation and Chaos 18, 3101-3111.

Lu, J., Ho, D. \& Kurths, J. [2009a] "Consensus over directed static networks with arbitrary communication delays," Phys. Rev. E 80, 066121.

Lu, J., Ho, D. \& Wang, Z. [2009b] "Pinning stabilization of linearly coupled stochastic neural networks via minimum number of controllers," IEEE Trans. Neural Netw. 20, 1617-1629.

Lu, J., Ho, D. \& Wu, L. [2009c] "Exponential stabilization in switched stochastic dynamical networks," Nonlinearity 22, 889-911.

Lu, J., Ho, D. \& Cao, J. [2010] "A unified synchronization criterion for impulsive dynamical networks," Automatica 46, 1215-1221.

Lu, J. \& Ho, D. [2011] "Stabilization of complex dynamical networks with noise perturbation under performance constraint," Nonlin. Anal.: Real World Appl. 12, 1974-1984.

Lu, J., Ho, D., Cao, J. \& Kurths, J. [2011] "Exponential synchronization of linearly coupled neural networks with impulsive disturbances," IEEE Trans. Neural Netw. 22, 329-335.

Mazenc, F., Malisoff, M. \& Harmand, J. [2008] "Further results on stabilization of periodic trajectories for a chemostat with two species," IEEE Trans. Autom. Contr. 53, 66-74.

Motter, A., Zhou, C. \& Kurths, J. [2005] "Enhancing complex-network synchronization," Europhys. Lett. 69, 334-340. 
Newman, M. \& Watts, D. [1999] "Scaling and percolation in the small-world network model," Phys. Rev. E 60, 7332-7342.

Olfati-Saber, R. \& Murray, R. [2004] "Consensus problems in networks of agents with switching topology and time-delays," IEEE Trans. Autom. Contr. 49, 1520-1533.

Ponce, C. M., Masoller, C. \& Martí, A. [2009] "Synchronizability of chaotic logistic maps in delayed complex networks," The Europ. Phys. J. B 67, 83-93.

Solé, R. \& Bascompte, J. [2006] Self-Organization in Complex Ecosystems (Princeton University Press).

Sorrentino, F., di Bernardo, M., Garofalo, F. \& Chen, G. [2007] "Controllability of complex networks via pinning," Phys. Rev. E 75, 046103.

Strogatz, S. [2001] "Exploring complex networks," Nature 410, 268-276.

Tang, Y., Leung, S., Wong, W. \& Fang, J. [2010] "Impulsive pinning synchronization of stochastic discretetime networks," Neurocomputing 73, 2132-2139.

Tang, Y., Wong, W., Fang, J. \& Miao, Q. [2011] "Pinning impulsive synchronization of stochastic delayed coupled networks," Chinese Phys. B 20, 040513.

Wang, L., Dai, H., Dong, H., Cao, Y. \& Sun, Y. [2008] "Adaptive synchronization of weighted complex dynamical networks through pinning," The Europ. Phys. J. B 61, 335-342.

Wang, X. \& Chen, G. [2002] "Synchronization in smallworld dynamical networks," Int. J. Bifurcation and Chaos 12, 187-192.

Wang, Z., Wang, Y. \& Liu, Y. [2010] "Global synchronization for discrete-time stochastic complex networks with randomly occurred nonlinearities and mixed time delays," IEEE Trans. Neural Netw. 21, 11-25.

Wu, C. [2007] Synchronization in Complex Networks of Nonlinear Dynamical Systems (World Scientific, Singapore).

Yang, T. [2001] Impulsive Systems and Control: Theory and Application (Nova Science, NY).

Yang, Z. \& Xu, D. [2007] "Stability analysis and design of impulsive control systems with time delay," IEEE Trans. Autom. Contr. 52, 1448-1454.

Yu, W., Chen, G. \& Lü, J. [2009] "On pinning synchronization of complex dynamical networks," Automatica 45, 429-435.

Zhang, H., Ma, T., Huang, G. \& Wang, Z. [2010] "Robust global exponential synchronization of uncertain chaotic delayed neural networks via dual-stage impulsive control," IEEE Trans. Syst. Man Cybern. Part B 40, 831-844.

Zhang, Q., Lu, J., Lu, J. \& Tse, C. [2008] "Adaptive feedback synchronization of a general complex dynamical network with delayed nodes," IEEE Trans. Circuits Syst.-II 55, 183-187.
Zhou, C., Motter, A. \& Kurths, J. [2006] "Universality in the synchronization of weighted random networks," Phys. Rev. Lett. 96, 034101.

\section{Appendix}

\section{Proof of Theorem 1}

Proof. Construct a Lyapunov function in the form of

$$
V(t)=\sum_{i=1}^{N} e_{i}^{T}(t) e_{i}(t) .
$$

For any $t \in\left[t_{k-1}, t_{k}\right)$, taking derivative of $V(t)$ along the solution of (6) gives that

$$
\begin{aligned}
D^{+} V(t)= & \sum_{i=1}^{N} 2\left[-e_{i}^{T}(t) C e_{i}(t)+e_{i}^{T}(t) B f_{1}\left(e_{i}(t)\right)\right) \\
& \left.+e_{i}^{T}(t) D f_{2}\left(e_{i}(t-\tau(t))\right)\right] \\
& +\sum_{i=1}^{N} \sum_{j=1}^{N} 2 c a_{i j} e_{i}^{T}(t) \Gamma H\left(e_{j}(t)\right) .
\end{aligned}
$$

Letting $d=l_{2} \sqrt{\lambda_{\max }\left(D^{T} D\right)}$, by Assumption 1, we obtain

$$
\begin{aligned}
& 2 e_{i}^{T}(t) B f_{1}\left(e_{i}(t)\right) \\
& \quad \leq 2\left\|e_{i}(t)\right\| \cdot \sqrt{\left\|B f_{1}\left(e_{i}(t)\right)\right\|^{2}} \\
& \quad \leq 2\left\|e_{i}(t)\right\| \cdot \sqrt{\lambda_{\max }\left(B^{T} B\right) l_{1}^{2}\left\|e_{i}(t)\right\|^{2}} \\
& \quad \leq 2 \sqrt{\lambda_{\max }\left(B^{T} B\right)} l_{1} e_{i}^{T}(t) e_{i}(t)
\end{aligned}
$$

and

$$
\begin{aligned}
2 e_{i}^{T}(t) D & f_{2}\left(e_{i}(t-\tau(t))\right) \\
\leq 2 & \cdot \sqrt{\frac{d}{\sqrt{\rho}}\left\|e_{i}(t)\right\|^{2}} \cdot \sqrt{\frac{\sqrt{\rho}}{d}\left\|D f_{2}\left(e_{i}(t-\tau(t))\right)\right\|^{2}} \\
=2 & \cdot \sqrt{\frac{d}{\sqrt{\rho}}\left\|e_{i}(t)\right\|^{2}} \\
& \cdot \sqrt{\frac{\sqrt{\rho}}{d} f_{2}^{T}\left(e_{i}(t-\tau(t))\right) D^{T} D f_{2}\left(e_{i}(t-\tau(t))\right)}
\end{aligned}
$$




$$
\begin{aligned}
& \leq 2 \cdot \sqrt{\frac{d}{\sqrt{\rho}}\left\|e_{i}(t)\right\|^{2}} \\
& \cdot \sqrt{\frac{\sqrt{\rho}}{d} \lambda_{\max }\left(D^{T} D\right) l_{2}^{2}\left\|e_{i}(t-\tau(t))\right\|^{2}} \\
& =2 \cdot \sqrt{\frac{d}{\sqrt{\rho}}\left\|e_{i}(t)\right\|^{2}} \cdot \sqrt{d \sqrt{\rho}\left\|e_{i}(t-\tau(t))\right\|^{2}} \\
& \leq \frac{d}{\sqrt{\rho}}\left\|e_{i}(t)\right\|^{2}+d \sqrt{\rho}\left\|e_{i}(t-\tau(t))\right\|^{2} .
\end{aligned}
$$

Let $e^{\iota}(t)=\left(e_{1 \iota}(t), e_{2 \iota}(t), \ldots, e_{N \iota}(t)\right)^{T}$ and $H\left(e^{\iota}(t)\right)=\left(h\left(e_{1 \iota}(t)\right), h\left(e_{2 \iota}(t)\right), \ldots, h\left(e_{N \iota}(t)\right)\right)^{T}$.

From the diffusivity property of matrix $A$ and the assumption of $[(h(u)-h(v)) /(u-v)] \geq \vartheta>0$, it follows that

$$
\begin{aligned}
\sum_{i=1}^{N} \sum_{j=1}^{N} 2 c a_{i j} e_{i}^{T}(t) \Gamma H\left(e_{j}(t)\right) & \\
= & \sum_{i=1}^{N} \sum_{j=1}^{N} 2 c a_{i j}\left[\sum_{\iota=1}^{n} e_{i \iota}(t) \gamma_{\iota} H\left(e_{j \iota}(t)\right)\right] \\
= & \sum_{\iota=1}^{n} 2 c \gamma_{\iota}\left[\sum_{i=1}^{N} \sum_{j=1}^{N} e_{i \iota}(t) a_{i j} H\left(e_{j \iota}(t)\right)\right] \\
= & \sum_{\iota=1}^{n} 2 c \gamma_{\iota}\left(e^{\iota}(t)\right)^{T} A H\left(e^{\iota}(t)\right) \\
= & -\sum_{\iota=1}^{n} c \gamma_{\iota} \sum_{i=1}^{N} \sum_{j=1, j \neq i}^{N} a_{i j}\left(e_{i \iota}(t)-e_{j \iota}(t)\right) \\
& \times\left(H\left(e_{i \iota}(t)\right)-H\left(e_{j \iota}(t)\right)\right) \\
\leq & -\sum_{\iota=1}^{n} \sum_{i=1}^{N} \sum_{j=1, j \neq i}^{N} \vartheta c \gamma_{\iota} a_{i j}\left(e_{i \iota}(t)-e_{j \iota}(t)\right)^{2} \\
\leq & 0 .
\end{aligned}
$$

Considering the inequalities (A.3)-(A.5), it follows from (A.2) that

$$
\begin{aligned}
D^{+} V(t) \leq & \sum_{i=1}^{N}\left[-2 e_{i}^{T}(t) C e_{i}(t)\right. \\
& \left.+2 \sqrt{\lambda_{\max }\left(B^{T} B\right)} l_{1} e_{i}^{T}(t) e_{i}(t)\right]
\end{aligned}
$$

$$
\begin{aligned}
& +\sum_{i=1}^{N} \frac{d}{\sqrt{\rho}}\left\|e_{i}(t)\right\|^{2} \\
& +\sum_{i=1}^{N} d \sqrt{\rho}\left\|e_{i}(t-\tau(t))\right\|^{2} \\
\leq & \sum_{i=1}^{N}\left(\alpha+\frac{d}{\sqrt{\rho}}\right) e_{i}^{T}(t) e_{i}(t) \\
& +\sum_{i=1}^{N} d \sqrt{\rho}\left\|e_{i}(t-\tau(t))\right\|^{2} \\
= & \left(\alpha+\frac{d}{\sqrt{\rho}}\right) V(t) \\
& +d \sqrt{\rho} V(t-\tau(t)),
\end{aligned}
$$

where $\alpha=\lambda_{\max }\left(-C-C^{T}\right)+2 \sqrt{\lambda_{\max }\left(B^{T} B\right)} l_{1}$.

When $t=t_{k}$, we have

$$
\begin{aligned}
V\left(t_{k}^{+}\right)= & \sum_{i=1}^{N} e_{i}^{T}\left(t_{k}^{+}\right) e_{i}\left(t_{k}^{+}\right) \\
= & \sum_{i \in \mathfrak{D}\left(t_{k}\right)}(1+\mu)^{2} e_{i}^{T}\left(t_{k}^{-}\right) e_{i}\left(t_{k}^{-}\right) \\
& +\sum_{i \notin \mathfrak{D}\left(t_{k}\right)} e_{i}^{T}\left(t_{k}^{-}\right) e_{i}\left(t_{k}^{-}\right) .
\end{aligned}
$$

Let $\varphi\left(t_{k}^{-}\right)=\min \left\{\left\|e_{i}\left(t_{k}^{-}\right)\right\|: i \in \mathfrak{D}\left(t_{k}^{-}\right)\right\}$and $\psi\left(t_{k}^{-}\right)=\max \left\{\left\|e_{i}\left(t_{k}^{-}\right)\right\|: i \notin \mathfrak{D}\left(t_{k}^{-}\right)\right\}$, then we can get $\psi\left(t_{k}^{-}\right) \leq \varphi\left(t_{k}^{-}\right)$.

Since $\rho=\frac{N+l \mu(\mu+2)}{N} \in(0,1)$, one has $N-l=$ $\frac{\left[\rho-(1+\mu)^{2}\right] l}{(1-\rho)} \geq 0$, which further gives that

$$
\begin{aligned}
& \sum_{i \notin \mathfrak{D}\left(t_{k}\right)} e_{i}^{T}\left(t_{k}^{-}\right) e_{i}\left(t_{k}^{-}\right) \\
& \leq(N-l) \psi^{2}\left(t_{k}^{-}\right) \leq(N-l) \varphi^{2}\left(t_{k}^{-}\right) \\
& \leq \frac{\left[\rho-(1+\mu)^{2}\right] l}{(1-\rho)} \varphi^{2}\left(t_{k}^{-}\right) \\
& \leq \frac{\rho-(1+\mu)^{2}}{(1-\rho)} \sum_{i \in \mathfrak{D}\left(t_{k}\right)} e_{i}^{T}\left(t_{k}^{-}\right) e_{i}\left(t_{k}^{-}\right) .
\end{aligned}
$$


Then by some calculations, we have

$$
\begin{gathered}
(1+\mu)^{2} \sum_{i \in \mathfrak{D}\left(t_{k}\right)} e_{i}^{T}\left(t_{k}^{-}\right) e_{i}\left(t_{k}^{-}\right)+\sum_{i \notin \mathfrak{D}\left(t_{k}\right)} e_{i}^{T}\left(t_{k}^{-}\right) e_{i}\left(t_{k}^{-}\right) \\
\leq \rho \sum_{i=1}^{N} e_{i}^{T}\left(t_{k}^{-}\right) e_{i}\left(t_{k}^{-}\right)=\rho V\left(t_{k}^{-}\right) .
\end{gathered}
$$

If follows from (A.7) that

$$
V\left(t_{k}^{+}\right) \leq \rho V\left(t_{k}^{-}\right)
$$

For any $\epsilon>0$, let $\nu(t)$ be a unique solution of the following impulsive delayed systems (A.11):

$$
\left\{\begin{aligned}
\dot{\nu}(t)= & \left(\alpha+\frac{d}{\sqrt{\rho}}\right) V(t)+d \sqrt{\rho} \cdot \nu(t-\tau(t)) \\
& +\epsilon, \quad t \geq 0, \quad t \neq t_{k}, \quad k \in \mathbb{N}, \\
\nu\left(t_{k}^{+}\right)=\rho \nu\left(t_{k}^{-}\right), \quad k \in \mathbb{N}, & \\
\nu(t)= & \sum_{i=1}^{N}\left\|\phi_{i}(t)\right\|^{2}, \quad-\tau^{*} \leq t \leq 0 .
\end{aligned}\right.
$$

According to Lemma 1, we have $\nu(t) \geq V(t) \geq$ 0 for any $t \geq 0$.

By using the formula for the variation of parameters [Lakshmikantham et al., 1989], we can obtain the following integral equation for $\nu(t)$ :

$$
\begin{aligned}
\nu(t)= & W(t, 0) \nu(0) \\
& +\int_{0}^{t} W(t, s)[d \sqrt{\rho} \cdot \nu(s-\tau(s))+\epsilon] d s, \\
t & \geq 0,
\end{aligned}
$$

where $W(t, s)(t>s \geq 0)$ is the Cauchy matrix of the following linear impulsive system:

$$
\begin{cases}\dot{w}(t)=\left(\alpha+\frac{d}{\sqrt{\rho}}\right) w(t), & t \geq 0, \quad t \neq t_{k}, \quad k \in \mathbb{N} \\ w\left(t_{k}^{+}\right)=\rho w\left(t_{k}^{-}\right), & k \in \mathbb{N} .\end{cases}
$$
we have

Since $0<\rho<1$ and $T=\max _{k \in \mathbb{N}}\left\{t_{k}-t_{k-1}\right\}$,

$$
\begin{aligned}
W(t, s) & =e^{\left(\alpha+\frac{d}{\sqrt{\rho}}\right)(t-s)} \prod_{s<t_{k} \leq t} \rho \\
& \leq e^{\left(\alpha+\frac{d}{\sqrt{\rho}}\right)(t-s)} \rho^{\left[\frac{(t-s)}{T}-1\right]}
\end{aligned}
$$

$$
\begin{array}{r}
=\rho^{-1} \cdot e^{\left(\alpha+\frac{d}{\sqrt{\rho}}+\frac{\ln \rho}{T}\right)(t-s)}, \\
t \geq s \geq 0 .
\end{array}
$$

Letting $\theta=\rho^{-1} \sup _{-\tau^{*} \leq s \leq 0}\left\{\sum_{i=1}^{N}\left\|\phi_{i}(s)\right\|^{2}\right\}$ and $\beta=-\left(\alpha+\frac{d}{\sqrt{\rho}}+\frac{\ln \rho}{T}\right)$, we have

$$
\begin{aligned}
\nu(t) \leq & \rho^{-1} \sum_{i=1}^{N}\left\|\phi_{i}(0)\right\|^{2} \cdot e^{\left(\alpha+\frac{d}{\sqrt{\rho}}+\frac{\ln \rho}{T}\right) t} \\
& +\int_{0}^{t} \rho^{-1} e^{\left(\alpha+\frac{d}{\sqrt{\rho}}+\frac{\ln \rho}{T}\right)(t-s)} \\
& \times[d \sqrt{\rho} \cdot \nu(s-\tau(s))+\epsilon] d s \\
\leq & \theta \cdot e^{-\beta t}+\int_{0}^{t} e^{-\beta(t-s)} \\
& \times\left[\rho^{-\frac{1}{2}} d \cdot \nu(s-\tau(s))+\rho^{-1} \epsilon\right] d s \\
& t \geq 0 .
\end{aligned}
$$

Let $g(\lambda)=\lambda-\beta+\rho^{-\frac{1}{2}} d \cdot e^{\lambda \tau^{*}}$. Since $\beta>0$, $d>0,0<\rho<1$ and $\alpha+\frac{2 d}{\sqrt{\rho}}+\frac{\ln \rho}{T}<0$, we have $g(0)=-\beta+\rho^{-\frac{1}{2}} d<0, g(\infty)>0$ and $g^{\prime}(\lambda)=1+\lambda \rho^{-\frac{1}{2}} d \cdot e^{\lambda \tau^{*}}>0$. Consequently, we can conclude that $g(\lambda)=0$ has a unique solution $\lambda>0$.

Since $0<\rho<1$, for $-\tau^{*} \leq t \leq 0$, we have

$$
\begin{aligned}
\nu(t) & \leq \rho^{-1} \cdot \sum_{i=1}^{N}\left\|\phi_{i}(t)\right\|^{2} \\
& <\theta e^{-\lambda t}+\frac{\epsilon}{\beta \rho-d \sqrt{\rho}} .
\end{aligned}
$$

In the following, we shall prove that, for $t \geq 0$, the following is true:

$$
\nu(t)<\theta e^{-\lambda t}+\frac{\epsilon}{\beta \rho-d \sqrt{\rho}} .
$$

If (A.17) is not true, then there exists a $t^{*}>0$ such that

$$
\nu\left(t^{*}\right) \geq \theta e^{-\lambda t^{*}}+\frac{\epsilon}{\beta \rho-d \sqrt{\rho}},
$$

and

$$
\nu(t)<\theta e^{-\lambda t}+\frac{\epsilon}{\beta \rho-d \sqrt{\rho}}, \quad \text { for } t<t^{*} .
$$


J. Lu et al.

By using (10), (A.15) and (A.19), some detailed calculations give that

$$
\begin{aligned}
\nu\left(t^{*}\right) & \leq \theta \cdot e^{-\beta t^{*}}+\int_{0}^{t^{*}} e^{-\beta\left(t^{*}-s\right)}\left[\rho^{-\frac{1}{2}} d \cdot \nu(s-\tau(s))+\rho^{-1} \epsilon\right] d s \\
& <e^{-\beta t^{*}}\left[\theta+\frac{\epsilon}{\beta \rho-d \sqrt{\rho}}+\int_{0}^{t^{*}} e^{\beta s}\left[\rho^{-\frac{1}{2}} d \cdot\left(\theta e^{-\lambda(s-\tau(s))}\right)+\rho^{-\frac{1}{2}} d \cdot \frac{\epsilon}{\beta \rho-d \sqrt{\rho}}+\rho^{-1} \epsilon\right] d s\right] \\
& \leq e^{-\beta t^{*}}\left[\theta+\frac{\epsilon}{\beta \rho-d \sqrt{\rho}}+\rho^{-\frac{1}{2}} d \theta e^{\lambda \tau^{*}} \int_{0}^{t^{*}} e^{(\beta-\lambda) s} d s+\frac{\beta \epsilon}{\beta \rho-d \sqrt{\rho}} \int_{0}^{t^{*}} e^{\beta s} d s\right] \\
& =\theta e^{-\beta t^{*}}+\frac{\epsilon}{\beta \rho-d \sqrt{\rho}} \cdot e^{-\beta t^{*}}+e^{-\beta t^{*}} \rho^{-\frac{1}{2}} d \theta e^{\lambda \tau^{*}} \cdot \frac{e^{(\beta-\lambda) t^{*}}-1}{\beta-\lambda}+e^{-\beta t^{*}} \cdot \frac{\beta \epsilon}{\beta \rho-d \sqrt{\rho}} \cdot \frac{e^{\beta t^{*}}-1}{\beta} \\
& =\theta e^{-\lambda t^{*}}+\frac{\epsilon}{\beta \rho-d \sqrt{\rho}},
\end{aligned}
$$

which leads to a contradiction with (A.18). Therefore, the inequality (A.18) holds. Letting $\epsilon \rightarrow 0$, for $t \geq 0$, we have

$$
\nu(t) \leq \theta e^{-\lambda t} .
$$

which further implies that $\sum_{i=1}^{N} e_{i}^{T}(t) e_{i}(t)=V(t) \leq \nu(t) \leq \theta e^{-\lambda t}$. The proof is hence completed by referring to Definition 3.1. 